\title{
A Review of Sensor Layout for Condition Monitoring during Discrete-part Manufacturing
}

\author{
Kang $\mathrm{He}^{1,2}$, Nan Wang ${ }^{1, *}$ and Lin $\mathrm{Zhu}^{3}$ \\ ${ }^{1}$ Mine Machinery and Electronic Eng. Research Center, Suzhou Univ.,Suzhou 234000,China \\ ${ }^{2}$ School of Mechanical Engineering, Southeast University, Nanjing 211189, China \\ ${ }^{3}$ Department of mechanical and aerospace eng. , Monash university, clayton3800, Australia \\ ${ }^{*}$ Corresponding author
}

\begin{abstract}
This paper presents in a unified way, the various strategies of optimal sensor placement for condition monitoring during discrete parts manufacturing. The objective of this paper, is to survey the current state of optimal sensor layout with two modules: sensor optimized layout for single target and sensor placement strategy under multi-targets and multi monitoring requirements. Each approach is outlined. Finally, the recommendations and challenges faced by industry and academia are discussed and several principle conclusions are drawn.
\end{abstract}

Keywords-sensor deployment; optimization; condition monitoring; discrete-part manufacturing

\section{INTRODUCTION}

The condition monitoring of manufacturing process is particularly important for improving the utilization ratio of machine tools, reducing the maintenance cost ${ }^{[1,2]}$, and ensuring the stability of product quality ${ }^{[3,4]}$. Condition monitoring in manufacturing process is to check and identify the function of operating equipment or parts. That is through analysis and processing the sensor data, then extracts feature related information to judge whether running condition has degradation sign, predict the degradation trend, confirm the wear degree ${ }^{[5,6]}$.

Condition monitoring in manufacturing processes is mainly to measure and assess some key parameters effectively ${ }^{[7,8]}$, which involves multi-sensor system inevitably. The intensive data provided by Sensor Network with different types, quantity, layout shows comprehensive description for dynamic change of manufacturing process state ${ }^{[1]}$. However, in the complex manufacturing system, sensor gather information is restricted by many aspects, such as the characteristics of itself, fault characteristics, processing technology, the layout of sensor and so on. Enough valuable information from sensor is benefit to improve the monitoring capability of system, but unplanned layout of sensor can not only increase the cost, but also reduce the monitoring and diagnosis efficiency ${ }^{[3]}$. In practice, redundant layout of sensor can not improve the monitoring ability, the system optimization target values has a maximum value with the increase of sensor quantity ${ }^{[9]}$, as shown in figure 1.1. In addition, the redundant sensor arrangement can reduce the loss of information effectively, but the vast amounts of data transmission not only need higher bandwidth, but also greatly increase the cost of data analysis and processing, which will especially true in the remote diagnosis and the wireless sensor network ${ }^{[10]}$. Therefore, the optimization sensor of layout, the type and the number in sensor system is very important.

For the diversity and complexity of the manufacturing system, and multiple layout algorithm of sensor, it will be not easy to optimize sensor layout. In order to know the sensor optimized layout technology at home and abroad, the research provides a detailed summary from the singularity and diversity of optimization objective.

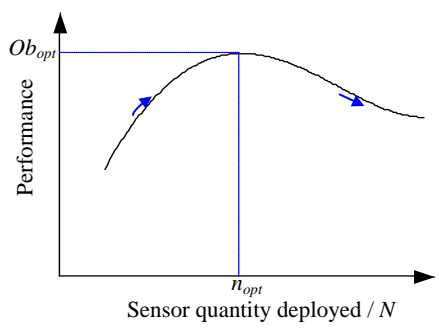

FIGURE I.SYSTEM PERFORMANCE VARIES WITH THE SENSOR QUANTITY DEPLOYED.

\section{Optimal SENSOR LAyOUT WITH SingLE TARgET}

Sensor deployment problem generally consists of four aspects, as illustrated in Fig. 2. This integer combinatorial optimization problem is built on the three types of sensor optimization problems ${ }^{[11]}$, which is usually an NP-hard problem. Therefore, computational intelligence plays an important role in solving such a problem. To this end, extensive and in-depth study is carried out by domestic and foreign scholars to optimize sensor layout for condition monitoring during manufacture ${ }^{[12-20]}$.

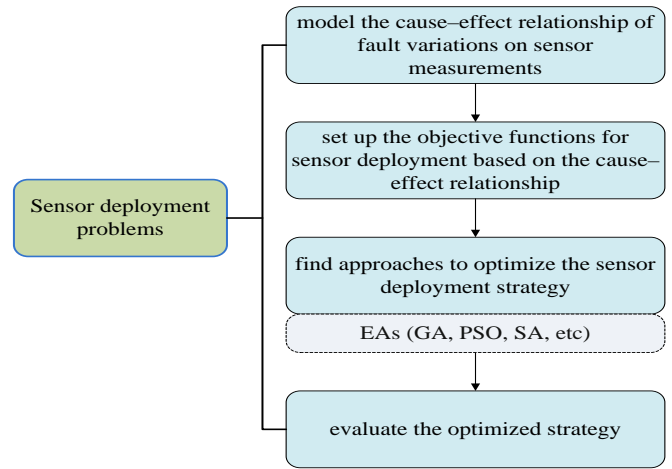

FIGURE II. PROCESS OF SOLVING THE OPTIMAL SENSOR PLACEMENT PROBLEM ${ }^{[21]}$. 
Based on the CAD data from the assembly process, $\operatorname{Khan}^{[12,22,23]}$ maximize the diagnosis vector model of the smallest single clamping apparatus to enhance the diagnosticability of multiple clamping apparatus fault during the stamped parts assembling process. Based on the digraph theory, Bhushan ${ }^{[13,24,25]}$ put forward a optimal layout on the fault monitoring system reliability maximization, which use the least price as the constraint condition to maximize the system reliability, But it only considers single-purpose optimization. Nof ${ }^{[14,26]}$ raise an economic principle of sensor optimal layout to realize the automation and quality control during the manufacturing processes, from which we get a general design criterion to optimize layout of sensor system. $\mathrm{Liu}^{[15,16]}$ use improved exchange algorithm and GA-oriented data mining algorithms to maximize sensitivity performance index during the sensor optimal layout, respectively, but which only consider the same type sensor. $\mathrm{Li}^{[17,20,27]}$ minimize system costs and meet diagnostic constraints by turning the problem of sensors arrangement to sensor coverage. $\mathrm{Cao}^{[18]}$ build nonlinear bearing model based on Jones' bearing theory, and increase the reliability of bearing fault diagnosis by optimizing sensor layout. Different with the method of diagnosing production error flow between the manufacture multi-station, BellanNebot ${ }^{[19]}$ using the effective independence method for singlestation fixture layout, but the initial layout of sensor depend on prior knowledge and can only meet the minimal optimization of sensor quantity.

Different with the above sensor optimized layouts meeting the diagnosticability target, Liu $^{[20,27,28]}$ put forward a targetoriented sensor layout strategy used for mean-shift monitoring and diagnosing, which build the causal relationship between a set of variables in distributed network based on BN, meeting the minimum cost in different ARL constraints and recognize the root cause in manufacturing process by partial sensor information. but it is single target optimization: the total price of minimum sensor layout.

In conclusion, the single-target optimal layout of sensor has been applied wildly for its Flexible form, but state dependent relation in manufacturing system, the single optimal target can hardly meet the monitoring requirement. Soit is necessary to conduct the deep study of sensor layout under the multi-target and multi-monitor condition ${ }^{[29]}$.

\section{Sensor Placement Strategy wiht Multitarget}

The fault identification ability of diagnosis system (fault isolation) or using the symptoms set represent the right degree of fault behavior (fault recognition), which can be used to evaluate the performance of the sensor layout as well. The spatial layout of sensor will be the only factor affecting the sensor network diagnostic performance under certain conditions, for example the same performance parameters conditions of sensor, the changeless of sensor types. Compared to the terminal detection and single fixture sensors layout based on the traditional discrete manufacturing process, the distributed multi-directional sensors layout based on discrete manufacturing system exhibits its features ${ }^{[12]}$, which has been got more and more attention. Figure 3 shows the differences and connections among the three layouts.
In general, there are numerous working steps and stations during the production process of products, so the monitoring reliability of which relay on the ability acquiring abnormal condition of distributed sensor ${ }^{[23]}$. The reliability of sensor detection system has different standards according different object, such as diagnosability, observability, uncertainty and so on. $\mathrm{Yu}^{[3,30,31]}$ propose a sensor layout method used for the multi-station assemble process. By building the state space model to fuse the sensor data, and then confirming the layout and quantity of sensor with a back propagation algorithm, which use the error propagation among station and error diagnosability in single-station as the indicators of sensor network performance For the partial observed discrete event systems, Jing ${ }^{[32]}$ raise a methods used to select the set of sensor optimizations, Which can provide sufficient information and meet the observability of smallest event. The method uses a bottom-up and top-down algorithm meeting polynomial complexity to optimize the set of sensor. But there are no concrete facts in industrial application. Sun ${ }^{[33]}$ research the impact of optimal sensor layout on the diagnosability of multistation manufacturing systems, defining the detectability, locatability, isolation as the three indicates, providing the twostep strategies of error propagation among stations and error diagnosability in single-station to diagnostic error source, confirming the type, location, and number of sensor with a heuristic back-propagation algorithm. But the algorithm do not take fault characteristic into account in the process of sensor arrangement.

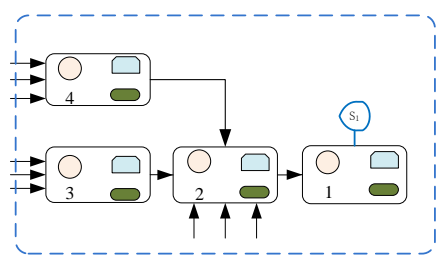

(A) Single fixture/station sensor layout

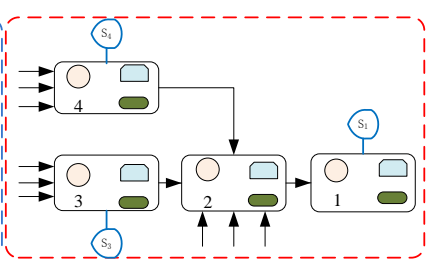

(B) Distributed sensor layout

\begin{tabular}{|c|c|c|}
\hline Items & A & B \\
\hline Th & $\begin{array}{l}\text { The } k \text { th fixture / processing / } \\
\text { assembly station }\end{array}$ & $\begin{array}{l}\text { The } k \text { th fixture / processing / } \\
\text { assembly station }\end{array}$ \\
\hline$\bigcirc \sqsubseteq$ & $\begin{array}{l}\text { Fixture / component movement } \\
\text { constraint }\end{array}$ & $\begin{array}{l}\text { Fixture / component movement } \\
\text { constraint }\end{array}$ \\
\hline $\mathrm{s}_{\mathrm{B}}$ The & e $k$ th fixture / station sensor & The $k$ th fixture / station sensor \\
\hline$\rightarrow$ & Component / information flow & Component / information flow \\
\hline Cost & Lower & Higher \\
\hline Layout features & Local / subsystem & Global / multi-station / multi-level \\
\hline $\begin{array}{l}\text { Monitoring } \\
\text { features }\end{array}$ & $\begin{array}{l}\text { Easy to quickly diagnose, } \\
\text { high precision }\end{array}$ & $\begin{array}{l}\text { High utilization of information, easy } \\
\text { to achieve multi-level diagnosis }\end{array}$ \\
\hline
\end{tabular}

FIGURE III. SENSOR SENSOR PLACEMENT FOR DISCRETE MANUFACTURING PROCESS

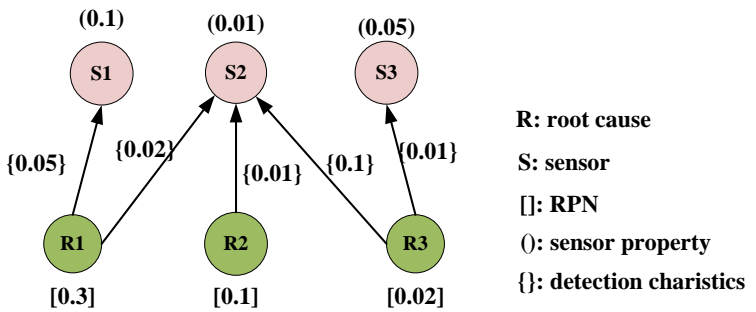

FIGURE IV. A FUZZY BIPARTITE GRAPH FOR SENSOR DEPLOYMENT. 
Avoided building the causal models between sensor and fault with complex formula, graphs theory is used for optimizing sensor placement because of the compactly ${ }^{[34]}$, comparing with truth-table, decision-tables and finite state model ${ }^{[35]} \cdot \mathrm{Wu}^{[1,34]}$ raise a sensor layout method based on the quantized fuzzy graph used for the fault diagnose in manufacturing system, as shown in figure 3 , In which building the causal models between sensor and faul based on the quantized fuzzy graph, setting the projection of various sensors and fault characteristic as unilateral value with analytic hierarchy process, then taking the minimum price of sensors and fault unobservable as the optimization goal, adopting the mixed integer linear programming model and greedy algorithm to optimize sensor layout. But the author does not indicate the impact of sensors and fault characteristic on the steady of system.

\section{Challenges AND OPPORTUNITIES}

The monitoring accuracy of sensor network is affected by many factors; Many layout strategies has got certain effects which focus on the algorithm implementation, which, But the study involved the sensor and fault mechanism are still in its infancy, or not understood fully. To this end, the main opportunities and challenges in manufacturing and academic interest lie in but not least.

(1) In theory, the monitoring ability can be improved by optimizing the sensor layout, but how to integrate sensor deployment strategy into diagnostics architecture to develop a real prototyping, so as to implement the diagnostic/prognostic algorithms more effectively and adjust the sensor deployment accordingly,is also need to be further studied.

(2)Whether the single-objective layout strategy or multiobjective layout strategy, the heterogeneous properties of the sensor and fault (such as failure rate, severity, etc.) have different effects on the system stability. Although this data can be obtained by appropriate statistical methods, the current optimal sensor layout is rarely discussed.

(3) Graph theory, as a more concise and compact expression, has been successfully used to model the causeeffect relationship between system faults and sensor measurements in the chemical process. But for discrete manufacturing process, how to make it apply to the sensor location still needs to be further explored.

(4) Although distributed sensor system has great advantages during status monitoring, the current study of which only stay at the system level. It is also an enormous challenge that how to integrate sensor and fault characteristic into the distributed sensor system.

\section{CONCLUSIONS AND DISCUSSION}

Optimal sensor deployment is an important research issue for condition monitoring during during manufacture. A lot of studies has been carried out and many great advances have been obtained. Overall, key information concerning optimal sensor deployment during manufacture is summarized as follows:
(1) For single-objective optimal strategy, more studies define the optimization objective as the single price optimization of sensor network, because of the diversity of monitoring requirement in manufacturing process, it can hardly meet the actual demand.

(2) As a compact expression, graph theory can describe the causal relationship between the sensor and fault efficiently, especially considering the self-characteristic of sensor and fault.

(3) Compared with single-objective layout strategy, multiobjective layout strategy mainly concentrate on the multiposition system, only considering the homogeneous sensor, lacking study on the characteristic of heterogeneous sensor and fault.

\section{ACKNOWLEDGMENT}

The authors would like to thank the National Natural Science Foundation of China (No. 51075070), the Natural Science Foundation of Anhui Province(No.1708085ME104), the Key Project of Natural Science Foundation of Universities of Anhui Province(No.KJ2017A439) , the Suzhou Univ. Professor $(\mathrm{PhD}) \quad$ Scientific Research Foundation (No.2016JB09), and the research platform open subject of Suzhou University(2015ykf23).

\section{REFERENCES}

[1] Z.Wu, S.J. Hsieh, J.Z.Li, “Sensor deployment based on fuzzy graph considering heterogeneity and multiple-objectives to diagnose manufacturing system,” Robot. Cim-int. Manuf. England, vol. 29, pp. 192-208, February 2013.

[2] Y. Lei, J. Lin, M. J. Zuo, Z. He, "Condition monitoring and fault diagnosis of planetary gearboxes: A review,” Measurement, vol.48, pp. 292-305.2014.

[3] Y. Ding, P. Kim, D. Ceglarek, J. Jin, “Optimal sensor distribution for variation diagnosis in multistation assembly processes," IEEE Rob. Autom Mag. vol.19, pp. 543-556, April 2003.

[4] Q. Ren, M. Balazinski, L. Baron, K. Jemielniak, R. Botez, "Type-2 fuzzy tool condition monitoring system based on acoustic emission in micromilling, ” INFORM SCIENCES, vol 255, pp. 121-134, 2014.

[5] AG. Rehorn, J. Jiang, PE. Orban, "State-of-the-art methods and results in tool condition monitoring: a review,” Int J Adv Manuf tech, vol.26, pp. 693-710, July -August 2005.

[6] W. Qiao, D. Lu, “A survey on wind turbine condition monitoring and fault diagnosis-Part I: Components and subsystems,” IEEE Ieee T Ind Electron, vol. 62, pp. 6536-6545, October 2015.

[7] H. Arslan, AO. Er, S. Orhan, E. Aslan, "Tool Condition Monitoring in Turning Using Statistical Parameters of Vibration Signal,” Int J Acoust Vib, vol. 21, pp. 371-378, April 2016.

[8] J. Guo, H. Jafarkhani, "Sensor deployment with limited communication range in homogeneous and heterogeneous wireless sensor networks," IEEE Trans. Wireless Commun,vol. 15, pp. 6771-6784, Ocboter 2016.

[9] M. Bhushan, R. Rengaswamy, "Design of sensor location based on various fault diagnostic observability and reliability criteria,” Comput Chem Eng, vol. 24, pp. 735-741, February 2000.

[10] IF. Akyildiz, W. Su, Y. Sankarasubramaniam, E. Cayirci, "Wireless sensor networks: a survey,” Comput Netw, vol. 38, pp. 393-422, April 2002.

[11] SS. Mandroli, AK. Shrivastava, Y. Ding, “A survey of inspection strategy and sensor distribution studies in discrete-part manufacturing processes,” Iie Trans, vol. 38, pp. 309-328, April 2006.

[12] A. Khan, D. Ceglarek, "Sensor optimization for fault diagnosis in multifixture assembly systems with distributed sensing," J Manuf Sci E-t Asme, vol. 122, pp. 215-226, January 2000. 
[13] M. Bhushan, R. Rengaswamy, "Comprehensive design of a sensor network for chemical plants based on various diagnosability and reliability criteria. 1. Framework,”Ind Eng Chem Res,vol. 41, pp. 18261839, July 2002.

[14] Y. Edan, SY. Nof, "Sensor economy principles and selection procedures,” IIE Trans, vol. 32, pp. 195-203, March 2000.

[15] CQ. Liu, Y. Ding, Y. Chen, "Optimal coordinate sensor placements for estimating mean and variance components of variation sources," IIE Trans, vol. 37, pp. 877-889, September 2005.

[16] Y. Ren, Y. Ding, "Optimal sensor distribution in multi-station assembly processes for maximal variance detection capability,” IIE Trans, vol. 41, pp. 804-818, September 2009.

[17] J. Li, J. Jin, "Optimal sensor allocation by integrating causal models and set-covering algorithms," IIE Trans, vol. 42, pp. 564-576, October 2010.

[18] H. Cao, L. Niu, Z. He, "Method for vibration response simulation and sensor placement optimization of a machine tool spindle system with a bearing defect,” Sens. Actuators, B, vol. 12, pp. 8732-8754, July 2012.

[19] JV. Abellan-Nebot, J. Liu, FR. Subirón, "Quality prediction and compensation in multi-station machining processes using sensor-based fixtures,” Robot Cim-Int Manuf, vol. 28, pp. 208-219, February 2012.

[20] K. Liu, J. Shi, "Objective-oriented optimal sensor allocation strategy for process monitoring and diagnosis by multivariate analysis in a Bayesian network,” Iie Trans, vol. 45, pp. 630-643, June 2013.

[21] Z. Wu, SJ. Hsieh, J. Li, "Sensor deployment based on fuzzy graph considering heterogeneity and multiple-objectives to diagnose manufacturing system,” Robot Cim-int Manuf, vol. 29, pp. 192-208, January 2013.

[22] A. Khan, D. Ceglarek, J. Shi, J. Ni, "Sensor optimization for fault diagnosis in single fixture systems: a methodology," J Manuf Sci E-T asme, vol 121, pp. 109-117, January 1999.

[23] A. Khan, D. Ceglarek, J. Ni, "Sensor location optimization for fault diagnosis in multi-fixture assembly systems,” J. Manuf. Sci. Eng, vol. 120, pp. 781-792, April 1998.

[24] VJ. Hodge, S. O'Keefe, M. Weeks, A. Moulds, "Wireless sensor networks for condition monitoring in the railway industry: A survey," IEEE Trans. Intell. Transp. Syst, vol. 16, pp.1088-1106, March 2015.

[25] M. Askarian, G. Escudero, M. Graells, R. Zarghami, "Fault diagnosis of chemical processes with incomplete observations: A comparative study," Comput Chem Eng, vol. 84, pp.104-116, 2016.

[26] H. Qi, S.S Iyengar, K. Chakrabarty, "Distributed sensor networks-a review of recent research,” J Franklin I, vol. 338, pp. 655-668, June 2001.

[27] MS. Sayed, N. Lohse, "Ontology-driven generation of Bayesian diagnostic models for assembly systems,” Int J Adv Manuf Tech, vol. 74, pp. 1033-1052, May-Ocboter 2014.

[28] Y. Liu, X. Ye, S. Jin, "A bayesian based process monitoring and fixture fault diagnosis approach in the auto body assembly process," Journal of Shanghai Jiaotong University (Science), vol. 21, pp. 164-172, February 2016.

[29] N. Shukla, D. Ceglarek, MK. Tiwari, "Key characteristics-based sensor distribution in multi-station assembly processes," J Intell Manuf, vol. 26, pp. 43-58, Juanay 2015.

[30] K. Bastani, Z. Kong, W. Huang, Y. Zhou, “Compressive sensing-based optimal sensor placement and fault diagnosis for multi-station assembly processes,” IIE Trans, vol. 48, pp. 462-474, May 2016.

[31] S. Tyagi, N. Shukla, S. Kulkarni, "Optimal design of fixture layout in a multi-station assembly using highly optimized tolerance inspired heuristic,”. Appl Math Model, vol. 40, pp. 6134-6147, November 2016.

[32] S. Jiang, R. Kumar, HE. Garcia, "Optimal sensor selection for discreteevent systems with partial observation,” IEEE T Automat Contr, vol. 48, pp. 369-381, March 2003.

[33] J. Sun, L. Xi, E. Pan, S. Du, T. Xia, "Design for diagnosability of multistation manufacturing systems based on sensor allocation optimization,” Comput Ind, vol. 60, pp. 501-509, July 2009.

[34] K. He, M. Jia, Q. Xu, "Optimal sensor deployment for manufacturing process monitoring based on quantitative cause-effect graph," IEEE Trans. Autom. Sci. Eng, vol. 13, pp. 963-975, February 2016.
[35] V. Venkatasubramanian, R. Rengaswamy, K. Yin, "A review of process fault detection and diagnosis: Part II: Qualitative models and search strategies,” Comput Chem Eng, vol. 27, pp. 313-326, March 2003. 